\author{
П. Г. Дегтяренко ${ }^{1}$, В. З. Грищак², д-р техн. наук, \\ Д. Д. Грищак \\ Н. Н. Дьяченко ${ }^{2}$ канд. ффиз.-мат. наук
}

\title{
ПОСТАНОВКА И ОСНОВНЫЕ РАЗРЕШАЮЩИЕ УРАВНЕНИЯ ЗАДАЧИ УСТОЙЧИВОСТИ ОБОЛОЧЕЧНОЙ КОНСТРУКЦИИ ТИПА «БОЧКА-ОЖИВАЛО» ПРИ ДЕЙСТВИИ ВНЕШНЕГО ДАВЛЕНИЯ
}

В данной работе формулируется задача устойчивости тонкостенной оболочечной конструкции типа «бочка-оживало» при наличии стыковочного шпангоута, находящейся под воздействием нормального внешнего давления. Получены основные разрешающие дифференциальные уравнения, позволяющие исследовать конструкцию с учетом радиуса кривизны образующей и жесткостных характеристик стыковочного шпангоута. Решение задачи строится на основе метода конечных разностей. Выявлено влияние относительной высоты подъема образующих и способов закрепления торцов на локальную и общую устойчивость отсеков. Указан подход к определению параметров равноустойчивости сопряженной конструкции, а также величины рациональной жесткости стыковочного шпангоута.

Ключевые слова: оболочечная конструкция типа «бочка-оживало», локальная и общая устойчивость, жесткость шпангоута, метод конечных разностей.

Введение. Вопросы рационального проектирования оболочечных систем и снижения их материалоемкости являются наиболее актуальными в конструкциях новой техники, в частности, авиационной, химиической, ракетно-космической и других отраслях промышленности. Особое внимание в последнее время направлено на изучение эффективных с точки зрения несущей способности и материалоемкости форм элементов конструкций, подверженных внешнему силовому воздействию [2, 9 -11]. Например, для авиационных конструкций и систем ракетно-космической техники представляется актуальным исследование гладких и подкрепленных ортогональной системой ребер оболочек вращения при наличии отсеков разной геометрической формы, наиболее эфффективных с точки зрения их устойчивости при воздействии внешних нагрузок и аэродинамических характеристик.

Данная работа посвящена формулировке постановки задачи и выводу основных уравнений устойчивости составной оболочечной системы типа «бочка-оживало». Основное внимание уделено определению критических нагрузок локальных и общих фрорм выпучивания при действии равномерного внешнего давления при наличии стыковочного шпангоута. Для учета изменения напряженно-деформированного состояния

() П. Г. Дегтяренко, В. 3. Грищак,

Д. Д. Грищак, Н. Н. Дьяченко, 2019 
конструкции при переходе через шпангоут использованы результаты исследований, приведенные в работах [3, 4, 6-8]. В соответствии с $[1,5]$, предполагается, что относительная подъемистость обоих отсеков конструкции составляет менее одной пятой наименьшего линейного ее размера, что позволило приближенно считать в качестве координатных линий общепринятые координатные линии для цилиндрической и конической оболочек соответственно.

Постановка задачи. Рассматривается упругая оболочечная конструкция кругового сечения постоянной толщины $h$, подкрепленная стыковочным шпангоутом (рис. 1). Конструкция состоит из двух отсеков бочкообразной и оживальной формы. Ставится задача о локальной и общей устойчивости конструкции, то есть устойчивости составляющих секций и конструкции в целом, подверженных равномерному внешнему давлению $q$. Отсеки рассматриваются как оболочки средней длины с ограничениями, описываемыми ниже. Для обеих секций ось вращения обозначена через $O z$. Координаты выбираются следующим образом: линейная меридианная координата (вдоль образующих цилиндра или конуса) для бочкообразного отсека обозначается $\bar{s}$, для оживального $s$; дуговая координата «бочки» - $y$, угловая координата в плоскости параллели «оживало» - $\varphi$.

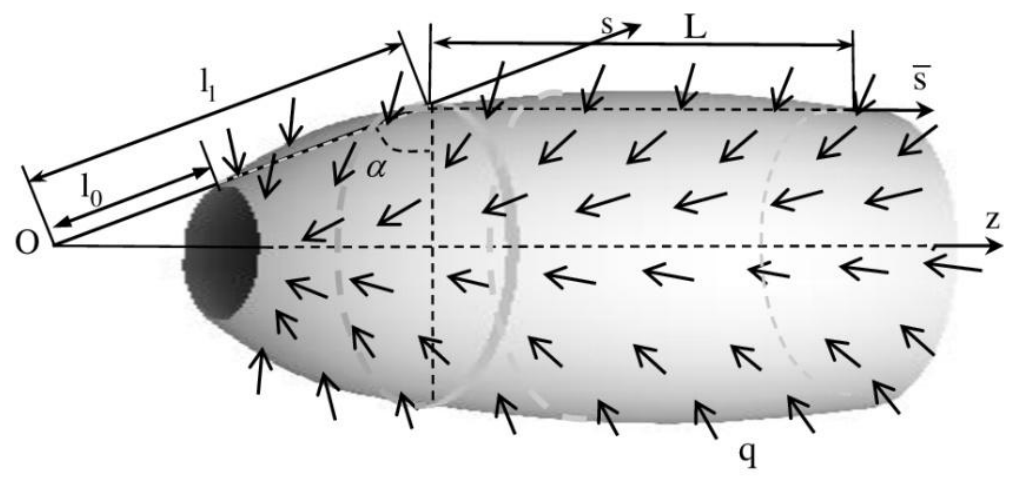

Рис. 1 - Схема оболочечной конструкции

Разрешающие уравнения устойчивости. Для вывода разрешающих уравнений устойчивости рассматриваемой конструкции формулируются уравнения поверхностей каждого отсека в предположении, что их подъемистость имеет синусоидальную форму.

Предполагается, что бочкообразная оболочка, как поверхность вращения, описывается следующей функцией радиуса параллельного круга в сечении, перпендикулярном оси вращения:

$$
r=R\left(1+C_{c y l} \sin \frac{\pi z}{L}\right),
$$


где $L$ и $R$ - расстояние между основаниями и радиус основания «бочки» соответственно, $C_{c y l}$ - относительная высота подъемистости «бочки».

На основании (1), учитывая, что в данной постановке $z=\bar{s}$, выписывается параметризация поверхности вращения:

$$
x=R\left(1+C_{c y l} \sin \frac{\pi \bar{s}}{L}\right) \cos \frac{y}{R} ; \quad y=R\left(1+C_{c y l} \sin \frac{\pi \bar{s}}{L}\right) \sin \frac{y}{R} ; \quad z=\bar{s} .
$$

В соответствии с введенным предположением, накладывается ограничение: $C_{c y l} \leq 1 / 5$. Для оболочки длины, удовлетворяющей неравенству $L / R \geq 1 / 2$, имеет место оценка $\lambda=R C_{c y l} \pi / L<1 / 3$. Тогда для всех $\bar{s} \in[0 ; L]$ в нулевом приближении можно записать:

$$
\begin{gathered}
\sqrt{1+\left(r^{\prime}\right)^{2}}= \\
=\sqrt{1+\left(R C_{c y l} \frac{\pi}{L} \cos \frac{\pi \bar{s}}{L}\right)^{2}}=1+\frac{1}{2}\left(\lambda \cos \frac{\pi \bar{s}}{L}\right)^{2}-\frac{1}{8}\left(\lambda \cos \frac{\pi \bar{s}}{L}\right)^{3}+\ldots \approx 1 .
\end{gathered}
$$

Радиусы кривизны с учетом (3) и соотношений, приведенных в [1, 5], принимаются в фрорме:

$$
\begin{gathered}
R_{1}=-\frac{\left(1+\left(r^{\prime}\right)^{2}\right)^{3 / 2}}{r^{\prime \prime}} \approx \frac{1}{R C_{c y l} \frac{\pi^{2}}{L^{2}} \sin \frac{\pi \bar{s}}{L}} \\
R_{2}=r \sqrt{1+\left(r^{\prime}\right)^{2}} \approx R\left(1+C_{c y l} \sin \frac{\pi \bar{s}}{L}\right) .
\end{gathered}
$$

Из (2) следует, что

$$
\begin{array}{lll}
\frac{\partial x}{\partial \bar{s}}=R C_{c y l} \frac{\pi}{L} \cos \frac{\pi \bar{s}}{L} \cos \frac{y}{R} ; & \frac{\partial y}{\partial s}=R C_{c y l} \frac{\pi}{L} \cos \frac{\pi \bar{s}}{L} \sin \frac{y}{R} ; & \frac{\partial z}{\partial \bar{s}}=1 ; \\
\frac{\partial x}{\partial y}=-\left(1+C_{c y l} \sin \frac{\pi \bar{s}}{L}\right) \sin \frac{y}{R} ; & \frac{\partial y}{\partial y}=\left(1+C_{c y l} \sin \frac{\pi \bar{s}}{L}\right) \cos \frac{y}{R} ; & \frac{\partial z}{\partial y}=0,
\end{array}
$$

откуда коэффицциенты первой квадратичной фрормы имеют вид:

$$
a_{11}=A_{1}^{2}=\left(\frac{\partial x}{\partial \bar{s}}\right)^{2}+\left(\frac{\partial y}{\partial \bar{s}}\right)^{2}+\left(\frac{\partial z}{\partial \bar{s}}\right)^{2}=1+\left(R C_{c y l} \frac{\pi}{L} \cos \frac{\pi \bar{s}}{L}\right)^{2} \approx 1 ;
$$




$$
\begin{gathered}
a_{12}=\frac{\partial x}{\partial \bar{s}} \frac{\partial x}{\partial y}+\frac{\partial y}{\partial s} \frac{\partial y}{\partial y}+\frac{\partial z}{\partial \bar{s}} \frac{\partial z}{\partial y}=0 \\
a_{22}=A_{2}^{2}=\left(\frac{\partial x}{\partial y}\right)^{2}+\left(\frac{\partial y}{\partial y}\right)^{2}+\left(\frac{\partial z}{\partial y}\right)^{2}=\left(1+C_{c y l} \sin \frac{\pi \bar{s}}{L}\right)^{2},
\end{gathered}
$$

тогда

$$
A_{1}=\sqrt{a_{11}} \approx 1 ; \quad A_{2}=\sqrt{a_{22}}=1+C_{c y l} \sin \frac{\pi \bar{s}}{L} .
$$

Применяя фрормулы, приведенные в [5], определяются фрункции распределения напряжений в основном докритическом безмоментном состоянии:

$$
\begin{gathered}
N_{10} \approx \frac{\sqrt{1+\left(r^{\prime}\right)^{2}}}{r} \int_{0}^{\bar{s}}(-q) r r^{\prime} d \bar{s} \approx \\
\approx-\frac{1}{R\left(1+C_{c y l} \sin \frac{\pi \bar{s}}{L}\right)} \int_{0}^{\bar{s}} q R^{2}\left(1+C_{c y l} \sin \frac{\pi \bar{s}}{L}\right) C_{c y l} \frac{\pi}{L} \cos \frac{\pi \bar{s}}{L} d \bar{s}= \\
=-q R C_{c y l} \sin \frac{\pi \bar{s}}{L} \cdot \frac{2+C_{c y l} \sin \frac{\pi \bar{s}}{L}}{2\left(1+C_{c y l} \sin \frac{\pi \bar{s}}{L}\right)} \approx-q R C_{c y l} \sin \frac{\pi \bar{s}}{L} ; \\
N_{20} \approx-\frac{r r^{\prime \prime}}{1+\left(r^{\prime}\right)^{2}} N_{10}+(-q) r \sqrt{1+\left(r^{\prime}\right)^{2}} \approx-r r^{\prime \prime} N_{10}-r q= \\
=-q R\left(1+C_{c y l} \sin \frac{\pi \bar{s}}{L}\right)\left[\left(R C_{c y l} \frac{\pi}{L} \sin \frac{\pi \bar{s}}{L}\right)^{2}+1\right] \approx-q R\left(1+C_{c y l} \sin \frac{\pi \bar{s}}{L}\right) .
\end{gathered}
$$

Для оживальной оболочки вводятся следующие обозначения (рис. 1): $l_{0}$ и $l_{1}$ - расстояние вдоль оси $O s$ до меньшего и большего оснований «оживало» соответственно; $\alpha$ - угол между осями $O s$ и $\mathrm{Oz}$. Предполагается, что оживальная оболочка, как поверхность вращения, описывается следующей функцией радиуса параллельного круга в сечении, перпендикулярном оси вращения:

$$
r(z)=z \operatorname{ctg} \alpha+C_{\text {cone }} R_{1, \text { cone }} \sin \left[\frac{\pi}{l_{1}-l_{0}}\left(\frac{z}{\sin \alpha}-l_{0}\right)\right]
$$

где $C_{\text {cone }}$ - относительная высота подъемистости «оживало». Поскольку $z=s \sin \alpha, R_{1, \text { cone }}=l_{1} \cos \alpha$, то функция (8) приобретет вид: 


$$
r(s)=\cos \alpha\left(s+l_{1} C_{\text {cone }} \sin \frac{\pi\left(s-l_{0}\right)}{l_{1}-l_{0}}\right) .
$$

Параметризация поверхности вращения представляется в форме:

$$
\begin{aligned}
& x=\cos \alpha\left(s+l_{1} C_{\text {cone }} \sin \frac{\pi\left(s-l_{0}\right)}{l_{1}-l_{0}}\right) \cos \varphi \\
& y=\cos \alpha\left(s+l_{1} C_{\text {cone }} \sin \frac{\pi\left(s-l_{0}\right)}{l_{1}-l_{0}}\right) \sin \varphi ; \\
& z=s \sin \alpha .
\end{aligned}
$$

Вводится обозначение

$$
\Omega=\frac{\pi}{l_{1}-l_{0}}\left(\frac{z}{\sin \alpha}-l_{0}\right)=\frac{\pi\left(s-l_{0}\right)}{l_{1}-l_{0}} .
$$

В соответствии с заданным предположением, подъемистость «оживало» удовлетворяет неравенству $R C_{\text {cone }}<1 / 5 \cdot R_{0}$, где $R_{0}=l_{0} \cos \alpha-$ радиус меньшего основания. Тогда $C_{\text {cone }} \leq l_{0} / 5 l_{1}$. Предположение $\frac{l_{0}}{l_{1}-l_{0}} \cos \alpha \leq \frac{1}{\pi}$ приводит к оценке

$$
t=C_{\text {cone }} \frac{\pi l_{1}}{l_{1}-l_{0}} \cos \alpha \leq \frac{\pi l_{0}}{5\left(l_{1}-l_{0}\right)} \cos \alpha \leq \frac{1}{5} .
$$

Тогда на основании представления (8) для всех $z \in\left[l_{0} \sin \alpha ; l_{1} \sin \alpha\right]$ в нулевом приближении получим

$$
\begin{gathered}
\sqrt{1+\left(\frac{d r}{d z}\right)^{2}}=\sqrt{1+\operatorname{ctg}^{2} \alpha\left(1+C_{\text {cone }} l_{1} \frac{\pi}{l_{1}-l_{0}} \cos \left[\frac{\pi}{l_{1}-l_{0}}\left(\frac{z}{\sin \alpha}-l_{0}\right)\right]\right)^{2}}= \\
=\frac{1}{\sin \alpha} \sqrt{1+\left(2 t \cos \Omega+t^{2} \cos ^{2} \Omega\right)}= \\
=\frac{1}{\sin \alpha}\left(1+\frac{1}{2}\left(2 t \cos \Omega+t^{2} \cos ^{2} \Omega\right)-\frac{1}{8}\left(2 t \cos \Omega+t^{2} \cos ^{2} \Omega\right)^{2}+\ldots\right) \approx \frac{1}{\sin \alpha} .
\end{gathered}
$$

Поэтому радиусы кривизны с учетом $[1,5]$ запишутся в виде:

$$
R_{1}=-\frac{\left(1+\left(r^{\prime}\right)^{2}\right)^{3 / 2}}{r^{\prime \prime}} \approx \frac{\left(l_{1}-l_{0}\right)^{2}}{C_{\text {cone }} l_{1} \pi^{2} \cos \alpha \sin \alpha \sin \Omega} ;
$$




$$
\begin{gathered}
R_{2}=r(z) \sqrt{1+\left(\frac{d r}{d z}\right)^{2}} \approx\left(z \operatorname{ctg} \alpha+C_{\text {cone }} l_{1} \cos \alpha \sin \Omega\right) \frac{1}{\sin \alpha}= \\
\operatorname{ctg} \alpha\left(s+C_{\text {cone }} l_{1} \sin \Omega\right) .
\end{gathered}
$$

Из параметризации (10) и представления (9) следует, что

$$
\begin{gathered}
\frac{\partial x}{\partial s}=\cos \alpha\left(1+\frac{\pi l_{1} C_{\text {cone }}}{l_{1}-l_{0}} \cos \Omega\right) \cos \varphi ; \frac{\partial x}{\partial \varphi}=-\cos \alpha\left(s+l_{1} C_{\text {cone }} \sin \Omega\right) \sin \varphi ; \\
\frac{\partial y}{\partial s}=\cos \alpha\left(1+\frac{\pi l_{1} C_{\text {cone }}}{l_{1}-l_{0}} \cos \Omega\right) \sin \varphi ; \frac{\partial y}{\partial \varphi}=\cos \alpha\left(s+l_{1} C_{\text {cone }} \sin \Omega\right) \cos \varphi ; \\
\frac{\partial z}{\partial s}=\sin \alpha, \frac{\partial z}{\partial \varphi}=0 .
\end{gathered}
$$

Коэфффициенты первой квадратичной формы в этом случае представляются в виде:

$$
\begin{gathered}
a_{11}=A_{1}^{2}=\left(\frac{\partial x}{\partial s}\right)^{2}+\left(\frac{\partial y}{\partial s}\right)^{2}+\left(\frac{\partial z}{\partial s}\right)^{2}=\sin ^{2} \alpha+\cos ^{2} \alpha\left(1+\frac{\pi l_{1} C_{\text {cone }}}{l_{1}-l_{0}} \cos \Omega\right)^{2} \approx 1 \\
a_{12}=0 ; \quad a_{22}=A_{2}^{2}=\left(\frac{\partial x}{\partial \varphi}\right)^{2}+\left(\frac{\partial y}{\partial \varphi}\right)^{2}+\left(\frac{\partial z}{\partial \varphi}\right)^{2}=\left(s+l_{1} C_{\text {cone }} \sin \Omega\right)^{2} .
\end{gathered}
$$

Таким образом,

$$
A_{1} \approx 1, \quad A_{2}=s+l_{1} C_{\text {cone }} \sin \Omega .
$$

Применяя формулы из [5], приближения (11) позволяют определить фрункции распределения напряжений в основном безмоментном состоянии:

$$
\begin{aligned}
& N_{10} \approx \frac{\sqrt{1+\left(r^{\prime}\right)^{2}}}{r} \int_{l_{0} \sin \alpha}^{z}(-q) r r^{\prime} d z=-\frac{q\left(z^{2}-l_{0}^{2} \sin ^{2} \alpha\right) \cos \alpha}{2 \sin ^{2} \alpha\left(z+C_{\text {cone }} l_{1} \sin \alpha \sin \Omega\right)}- \\
& -q C_{\text {cone }} l_{1} \operatorname{ctg} \alpha \sin \Omega \frac{\left(2 z+C_{\text {cone }} l_{1} \sin \alpha \sin \Omega\right)}{2\left(z+C_{\text {cone }} l_{1} \sin \alpha \sin \Omega\right)} \approx \\
& \approx-\frac{q\left(z^{2}-l_{0}^{2} \sin ^{2} \alpha\right) \cos \alpha}{2 \sin ^{2} \alpha\left(z+C_{\text {cone }} l_{1} \sin \alpha \sin \Omega\right)}-q C_{\text {cone }} l_{1} \operatorname{ctg} \alpha \sin \Omega ;
\end{aligned}
$$




$$
\begin{gathered}
N_{20} \approx-\frac{r r^{\prime \prime}}{1+\left(r^{\prime}\right)^{2}} N_{10}+(-q) r \sqrt{1+\left(r^{\prime}\right)^{2}} \approx-r r^{\prime \prime} N_{10} \sin ^{2} \alpha-\frac{r q}{\sin \alpha}= \\
=q\left(\frac{z \cos \alpha}{\sin ^{2} \alpha}+C_{\text {cone }} l_{1} \operatorname{ctg} \alpha \sin \Omega\left(1+\frac{\pi^{2} \operatorname{ctg}^{2} \alpha\left(z^{2}-l_{0}^{2} \sin ^{2} \alpha\right)}{2\left(l_{1}-l_{0}\right)^{2}}\right)\right)+ \\
+q\left(l_{1} C_{\text {cone }} \frac{\pi c t g \alpha}{l_{1}-l_{0}} \sin \Omega\right)^{2} \cos \alpha\left(z+C_{\text {cone }} l_{1} \sin \alpha \sin \Omega\right) .
\end{gathered}
$$

С учетом представления $z=s \sin \alpha$, внутренние усилия приобретут вид:

$$
\begin{gathered}
N_{10} \approx-\frac{q\left(s^{2}-l_{0}^{2}\right) \operatorname{ctg} \alpha}{2\left(s+C_{\text {cone }} l_{1} \sin \Omega\right)}-q C_{\text {cone }} l_{1} \operatorname{ctg} \alpha \sin \Omega ; \\
N_{20} \approx q\left(\frac{z \cos \alpha}{\sin ^{2} \alpha}+C_{\text {cone }} l_{1} \operatorname{ctg} \alpha \sin \Omega\left(1+\frac{\pi^{2} \operatorname{ctg} \alpha\left(z^{2}-l_{0}^{2} \sin ^{2} \alpha\right)}{2\left(l_{1}-l_{0}\right)^{2}}\right)\right) .
\end{gathered}
$$

Для получения разрешающих уравнений потребуются известные характеристики [1]:

- операторы дифреренцирования

$$
\begin{aligned}
& \nabla^{2}=\frac{1}{A_{1} A_{2}}\left(\frac{\partial}{\partial \bar{s}}\left(\frac{A_{2}}{A_{1}} \frac{\partial}{\partial \bar{s}}\right)+\frac{\partial}{\partial y}\left(\frac{A_{1}}{A_{2}} \frac{\partial}{\partial y}\right)\right) \\
& \nabla_{k}^{2}=\frac{1}{A_{1} A_{2}}\left(\frac{\partial}{\partial \bar{s}}\left(\frac{1}{R_{2}} \frac{A_{2}}{A_{1}} \frac{\partial}{\partial \bar{s}}\right)+\frac{\partial}{\partial y}\left(\frac{1}{R_{1}} \frac{A_{1}}{A_{2}} \frac{\partial}{\partial y}\right)\right) ;
\end{aligned}
$$

- формулы изменения кривизн срединной поверхности

$$
\begin{aligned}
& \kappa_{1}(\bar{s}, y)=-\frac{1}{A_{1}} \cdot \frac{\partial}{\partial \bar{s}}\left(\frac{1}{A_{1}} \cdot \frac{\partial w}{\partial \bar{s}}\right)-\frac{1}{\left(A_{2}\right)^{2} A_{1}} \cdot \frac{\partial A_{1}}{\partial y} \cdot \frac{\partial w}{\partial y} \\
& \kappa_{2}(\bar{s}, y)=-\frac{1}{A_{2}} \cdot \frac{\partial}{\partial y}\left(\frac{1}{A_{2}} \cdot \frac{\partial w}{\partial y}\right)-\frac{1}{\left(A_{1}\right)^{2} A_{2}} \cdot \frac{\partial A_{2}}{\partial \bar{s}} \cdot \frac{\partial w}{\partial \bar{s}}
\end{aligned}
$$

- фриктивная поперечная нагрузка

$$
g=-\left(N_{10} \kappa_{1}+N_{20} \kappa_{2}\right)
$$


- система основных уравнений в частных производных, характеризующая равновесие оболочки:

$$
\left\{\begin{array}{l}
D \nabla^{4} w-\nabla_{k}^{2} f=g \\
\frac{1}{E h} \nabla^{4} f+\nabla_{k}^{2} w=0
\end{array}\right.
$$

где $D=\frac{E h^{3}}{12\left(1-v^{2}\right)}-$ жесткость обшивки на изгиб; $E$ - модуль упругости и $v$ - коэффрициент Пуассона материала оболочки; $w$ - нормальное перемещение точки срединной поверхности при выпучивании; $f$ функция усилий.

Выписанные выше соотношения позволяют вывести разрешающие уравнения для оболочек «бочкообразной» и «оживальной» фрорм.

Для бочкообразной оболочки вводятся обозначения

$$
\varepsilon_{1}=\frac{h}{R}, K=\frac{L}{R}, \omega=12\left(1-v^{2}\right),
$$

безразмерные координаты $\bar{x}=\bar{s} / L, \bar{\varphi}=y / R$, безразмерное усилие

$$
\chi_{c y l}=\frac{q R^{2}}{E h^{2}} .
$$

Функцию радиальных перемещений и функцию усилий принимают в форме

$$
w_{c y l}(\bar{x}, \bar{\varphi})=W_{c y l}(\bar{x}) \cdot \cos (n \bar{\varphi}) ; \quad f_{c y l}(\bar{x}, \bar{\varphi})=E h^{2} \Phi_{c y l}(\bar{x}) \cdot \cos (n \bar{\varphi}) .
$$

С учетом (4) - (7), (17) - (20) после преобразований из (21) получаем систему:

$$
\begin{gathered}
\frac{\pi^{2} n^{2} C_{c y l}}{K^{2}} \sin \pi x\left(C_{c y l} \sin \pi x+1\right)^{2} \Phi_{c y l}(x)-\frac{1}{K^{2}}\left(C_{c y l} \sin \pi x+1\right)^{3} \Phi_{c y l}^{\prime \prime}(x)+ \\
+\left[\frac{\varepsilon_{1}}{\omega}\left(n^{2}+\frac{2}{K^{2}} \pi^{2} C_{c y l}\left(C_{c y l} \sin ^{2} \pi x-\sin \pi x-2 C_{c y l}\right)\right)-\right. \\
\left.-\left(C_{c y l} \sin \pi x+1\right)^{3} \chi_{c y l}\right] n^{2} W_{c y l}(x)+\pi C_{c y l} \cos \pi x\left(C_{c y l} \sin \pi x+1\right) W_{c y l}^{\prime}(x) \times
\end{gathered}
$$




$$
\begin{gathered}
\times\left[\frac{\chi_{c y l}}{K^{2}}\left(C_{c y l} \sin \pi x+1\right)^{3}+\frac{\varepsilon_{1}}{\omega K^{2}}\left(2 n^{2}+\frac{\pi^{2}\left(C_{c y l}^{2}-1\right)}{K^{4}}\right)\right]+ \\
+\frac{1}{K^{2}}\left(C_{c y l} \sin \pi x+1\right)^{2}\left[C_{c y l} \sin \pi x\left(C_{c y l} \sin \pi x+1\right)^{2} \chi_{c y l}-\right. \\
\left.-\frac{\varepsilon_{1}}{\omega}\left(2 n^{2}+\frac{\pi^{2}}{K^{2}} C_{c y l}\left(C_{c y l}\left(\sin ^{2} \pi x+1\right)+2 \sin \pi x\right)\right)\right] W_{c y l}^{\prime \prime}(x)+ \\
+\frac{\pi \varepsilon_{1}}{\omega K^{4}}\left(C_{c y l} \sin \pi x+1\right)^{3}\left[2 C_{c y l} \cos \pi x W_{c y l}^{\prime \prime \prime}(x)+\left(C_{c y l} \sin \pi x+1\right) W_{c y l}^{I V}(x)\right]=0 \\
\left(1+\frac{2}{K^{2} n^{2}} C_{c y l} \pi^{2}\left(C_{c y l}\left(\sin ^{2} \pi x-2\right)-\sin \pi x\right)\right) \Phi_{c y l}(x)+ \\
+C_{c y l} \pi \cos \pi x\left(C_{c y l} \sin \pi x+1\right)\left(\frac{2}{K^{2} n^{2}}+\frac{\pi^{2}}{K^{4} n^{4}}\left(C_{c y l}^{2}-1\right)\right) \Phi_{c y l}^{\prime}(x)- \\
-\left(C_{c y l} \sin \pi x+1\right)^{2}\left(\frac{2}{K^{2} n^{2}}+\frac{\pi^{2}}{K^{4} n^{4}} C_{c y l}\left(C_{c y l}\left(\sin ^{2} \pi x+1\right)+2 \sin \pi x\right)\right) \Phi_{c y l}^{\prime \prime}(x)+ \\
+\frac{2 \pi C_{c y l}}{K^{4} n^{4}} \cos \pi x\left(C_{c y l} \sin \pi x+1\right)^{3} \Phi_{c y l}^{\prime \prime \prime}(x)+\frac{1}{K^{4} n^{4}}\left(C_{c y l} \sin \pi x+1\right)^{4} \Phi_{c y l}^{I V}(x)- \\
-\frac{\pi^{2} C_{c y l}}{\varepsilon_{1} K^{2} n^{2}} \sin \pi x\left(C_{c y l} \sin \pi x+1\right)^{2} W_{c y l}(x)+\frac{1}{\varepsilon_{1} K^{2} n^{4}}\left(C_{c y l} \sin \pi x+1\right)^{3} W_{c y l}^{\prime \prime}(x)=0
\end{gathered}
$$

Поскольку для тонких оболочек средней длины $K^{2}>1, n^{2}>1$, то после исключения из второго уравнения слагаемых порядка, выше $\frac{1}{K^{2} n^{2}}$, преобразованное уравнение позволяет получить фрункцию $\Phi_{c y l}(x)$ в виде

$$
\Phi_{c y l}(x)=\frac{\left(C_{c y l} \sin \pi x+1\right)^{2}}{\varepsilon_{1} K^{2} n^{2}}\left[\pi^{2} C_{c y l} \sin \pi x W_{c y l}(x)-\frac{1}{n^{2}}\left(C_{c y l} \sin \pi x+1\right) W_{c y l}^{\prime \prime}(x)\right],
$$

подставляя которую в первое уравнение системы, получаем разрешающее диффреренциальное уравнение основного напряженного состояния относительно функции прогиба цилиндрической бочкообразной оболочки: 


$$
a_{4} W_{c y l}^{I V}(\bar{x})+a_{3} W_{c y l}^{\prime \prime \prime}(\bar{x})+a_{2} W_{c y l}^{\prime \prime}(\bar{x})+a_{1} W_{c y l}^{\prime}(\bar{x})+a_{0} W_{c y l}(\bar{x})=0,
$$

где

$$
\begin{aligned}
a_{0}= & \frac{2 \pi^{3} n^{2} \varepsilon_{1}^{2} C_{c y l}}{\omega K^{2}}\left(C_{c y l} \sin ^{2} \pi x-\sin \pi x-2 C_{c y l}\right)-\pi n^{2} \varepsilon_{1}\left(C_{c y l} \sin \pi x+1\right)^{3} \chi_{c y l}+ \\
+ & \frac{\pi n^{4} \varepsilon_{1}^{2}}{\omega}+\frac{\pi^{5}}{K^{4}} C_{c y l}\left(C_{c y l} \sin \pi x+1\right)^{3}\left[C_{c y l} \sin ^{2} \pi x\left(C_{c y l} \sin \pi x+1\right)+\right. \\
& \left.+\frac{1}{n^{2}}\left(9 C_{c y l}^{2} \sin ^{3} \pi x+8 C_{c y l} \sin ^{2} \pi x-6 C_{c y l}^{2} \sin \pi x+\sin \pi x-4 C_{c y l}\right)\right] \\
a_{1}= & \frac{\cos \pi x}{K^{2}}\left(C_{c y l} \sin \pi x+1\right)\left\{\varepsilon_{1} C_{c y l} \pi^{2}\left(C_{c y l} \sin \pi x+1\right)^{3} \chi_{c y l}+2 \varepsilon_{1}^{2} n^{2} \omega^{-1} C_{c y l} \pi^{2}+\right. \\
& \left.+\frac{\pi^{4}}{K^{2}} C_{c y l}\left[\varepsilon_{1}^{2} \omega^{-1}\left(C_{c y l}^{2}-1\right)-\frac{2}{n^{2}}\left(3 C_{c y l} \sin \pi x+1\right)\left(C_{c y l} \sin \pi x+1\right)^{3}\right]\right\} \\
a_{2}= & \frac{1}{K^{2}}\left(C_{c y l} \sin \pi x+1\right)^{2}\left\{\varepsilon_{1} C_{c y l} \pi \sin \pi x\left(C_{c y l} \sin \pi x+1\right)^{2} \chi_{c y l}-2 \varepsilon_{1} n^{2} \omega^{-1} \pi+\right. \\
+\frac{\pi^{3}}{K^{2}} & C_{c y l}\left[-\varepsilon_{1}^{2} \omega^{-1}\left(C_{c y l} \sin ^{2} \pi x+2 \sin \pi x+C_{c y l}\right)-\frac{2}{n^{2}} \sin \pi x\left(C_{c y l} \sin \pi x+1\right)^{3}-\right. \\
& \left.\left.-\frac{3}{n^{4}} \sin \pi x\left(3 C_{c y l} \sin ^{2} \pi x+\sin \pi x-2 C_{c y l}\right)\left(C_{c y l} \sin \pi x+1\right)^{2}\right]\right\} ; \\
a_{3}= & \frac{2 \pi^{2} C_{c y l}}{n^{4} K^{4}} \cos \pi x\left(C_{c y l} \sin ^{2} \pi x+1\right)^{3}\left(3 C_{c y l}^{2} \sin { }^{2} \pi x+6 C_{c y l} \sin \pi x+\frac{n^{4} \varepsilon_{1}^{2}}{\omega}+3\right) ; \\
a_{4}= & \frac{\pi}{n^{4} K^{4}}\left(C_{c y l} \sin ^{2} \pi x+1\right)^{4}\left(C_{c y l}^{2} \sin ^{2} \pi x+2 C_{c y l} \sin \pi x+\varepsilon_{1}^{2} \omega^{-1} n^{4}+1\right) .
\end{aligned}
$$

В предельном случае $C_{c y l}=0$ имеем уравнение для цилиндрической оболочки:

$$
\frac{1}{K^{4}}\left(\frac{\varepsilon_{1} n^{2}}{\omega}+\frac{1}{n^{2} \varepsilon_{1}}\right) W_{c y l}^{I V}(\bar{x})-\frac{2 n^{4}}{\omega K^{2}} W_{c y l}^{\prime \prime}(\bar{x})+n^{4}\left(\frac{n^{2} \varepsilon_{1}}{\omega}-\chi_{c y l}\right) W_{c y l}(\bar{x})=0,
$$

являющееся приближенным к уравнению, приведенному в [4].

Для оживальной оболочки (так же, как и для бочкообразной) вводятся обозначения: 


$$
\delta=\frac{n^{2}}{\cos ^{2} \alpha}, p=\varepsilon \delta ; \quad \varepsilon=\sqrt{\frac{h \operatorname{ctg} \alpha}{l_{1} \sqrt{12\left(1-v^{2}\right)}}}, \quad K_{c}=\frac{l_{0}}{l_{1}} ;
$$

- безразмерная координата $x=s / l_{1}$, безразмерное усилие

$$
\chi_{\text {cone }}=\frac{q^{*} l_{1}}{E h \varepsilon^{3} \operatorname{tg}^{3} \alpha} ;
$$

- функция радиальных перемещений и функция усилий

$$
\begin{gathered}
w_{\text {cone }}(x, \varphi)=W_{\text {cone }}(x) \operatorname{tg} \alpha \cdot \cos (n \varphi) ; \\
f_{\text {cone }}(x, \varphi)=\Phi_{\text {cone }}(x) \varepsilon^{2} l_{1} \text { Eh } \operatorname{tg}^{2} \alpha \cdot \cos (n \varphi),
\end{gathered}
$$

которые вместе с характеристиками (12) - -(16), (17) - (20) подставляются в систему (21). Поскольку для тонких оболочек средней длины $\varepsilon<<1, n^{2}>>1$, то можно удерживать в уравнениях слагаемые порядка, не выше $\varepsilon$. После некоторых преобразований получаем систему:

$$
\begin{gathered}
\varepsilon p \sin \alpha\left[-2 p\left(1-K_{c}\right)^{2}+\pi^{2}\left(C_{\text {cone }} \sin \Omega+x\right)^{2}\left(2 x\left(1-K_{c}\right)^{2}-\right.\right. \\
\left.-C_{\text {cone }} \sin \Omega\left(-2+\pi^{2} K_{c}^{2} \cos ^{2} \alpha+4 K_{c}-2 K_{c}^{2}-\pi^{2} x^{2} \cos ^{2} \alpha\right) \chi_{\text {cone }}\right] W_{\text {cone }}(x)- \\
-2 \pi^{2} p C_{\text {cone }} \cos ^{2} \alpha \sin \alpha \sin \Omega\left(C_{\text {cone }} \sin \Omega+x\right)^{2} \Phi_{\text {cone }}(x)+ \\
+2 \varepsilon \sin \alpha\left(1-K_{c}\right)^{2}\left(C_{\text {cone }} \sin \Omega+x\right)^{3} \Phi_{\text {cone }}^{\prime \prime}(x)=0 ; \\
p^{2} \varepsilon\left(1-K_{c}\right)^{2} \Phi_{\text {cone }}(x)-\pi^{2} p \cos ^{2} \alpha C_{\text {cone }} \sin \Omega\left(C_{\text {cone }} \sin \Omega+x\right)^{2} W_{\text {cone }}(x)+ \\
+\varepsilon\left(C_{\text {cone }} \sin \Omega+x\right)^{3}\left(1-K_{c}\right)^{2} W_{\text {cone }}^{\prime \prime}(x)=0 .
\end{gathered}
$$

Исключая из приведенной системы функцию $\Phi_{\text {cone }}(x)$, приходим к разрешающему дифференциальному уравнению для оживальной оболочки:

$$
a_{4} W_{\text {cone }}^{I V}(\bar{x})+a_{3} W_{\text {cone }}^{\prime \prime \prime}(\bar{x})+a_{2} W_{\text {cone }}^{\prime \prime}(\bar{x})+a_{1} W_{\text {cone }}^{\prime}(\bar{x})+a_{0} W_{\text {cone }}(\bar{x})=0,
$$

где

$$
\begin{aligned}
a_{0}= & \chi_{\text {cone }} p^{3}\left(C_{\text {cone }} \sin \Omega+x\right)^{2}\left(1-K_{c}\right)^{2}\left[\frac{1}{2} \pi^{2} \cos ^{2} \alpha C_{\text {cone }} \sin \Omega\left(K_{c}^{2}-x^{2}\right)-\right. \\
& \left.-\left(C_{\text {cone }} \sin \Omega+x\right)\left(1-K_{c}\right)^{2}\right]+\delta \pi^{2} \cos ^{2} \alpha C_{\text {cone }}\left(C_{\text {cone }} \sin \Omega+x\right)^{3} \times
\end{aligned}
$$




$$
\begin{gathered}
\times\left[-4 x \pi^{2} C_{\text {cone }}-4 \pi\left(2 C_{\text {cone }} \sin \Omega+x\right)\left(1-K_{c}\right) \cos \Omega+9 \pi^{2} C_{\text {cone }}^{2} \sin ^{3} \Omega+\right. \\
\left.+8 x \pi^{3} C_{\text {cone }} \sin ^{2} \Omega-\sin \Omega\left(\pi^{2}\left(6 C_{\text {cone }}^{2}-x^{2}\right)+2\left(1-K_{c}\right)^{2}\right)\right]- \\
-\delta^{2} \pi^{4} \cos ^{4} \alpha C_{\text {cone }}^{2} \sin ^{2} \Omega\left(C_{\text {cone }} \sin \Omega+x\right)^{4}+p^{4}\left(1-K_{c}\right)^{4} \\
a_{1}=-2 \delta \pi^{2} \cos ^{2} \alpha C_{\text {cone }}\left(C_{\text {cone }} \sin \Omega+x\right)^{4}\left(1-K_{c}\right) \times \\
\times\left[-2 K_{c} \sin \Omega+1,5 \pi C_{\text {cone }} \sin 2 \Omega+2 \sin \Omega+\pi x \cos \Omega\right] \\
+3 C_{\text {cone }}\left(-3 \pi^{2} C_{\text {cone }} \sin ^{2} \Omega-\pi^{2} x \sin \Omega+2 \pi^{2} C_{\text {cone }}-4 \pi K_{c} \cos \Omega+4 \pi \cos \Omega\right)- \\
\left.-2 \delta \pi^{2} \cos ^{2} \alpha C_{\text {cone }} \sin \Omega\left(C_{\text {cone }} \sin \Omega+x\right)\right\} \\
a_{3}=6\left(C_{\text {cone }} \sin \Omega+x\right)^{5}\left(1-K_{c}\right)^{3}\left(\pi C_{\text {cone }} \cos \Omega-K_{c}+1\right) \\
a_{4}=\left(C_{\text {cone }} \sin \Omega+x\right)^{6}\left(1-K_{c}\right)^{4} .
\end{gathered}
$$

В предельном случае $\left(C_{\text {cone }}=0\right)$ получаем уравнение для конической оболочки, приведенное в [1, 8]:

$$
W^{I V}+\frac{6}{x} W^{\prime \prime \prime}+\frac{6}{x^{2}} W^{\prime \prime}-\left(\frac{\chi p^{3}}{x^{3}}-\frac{p^{4}}{x^{6}}\right) W=0 .
$$

Метод решения. С целью согласования искомых критических давлений на «бочкообразном» и «оживальном» отсеках осуществляется обратный переход к размерному внешнему давлению $q$ с использованием формул (22) и (24).

Для численного решения задачи устойчивости исследуемой конструкции применяется метод конечных разностей, в котором дифференциальное уравнение сводится к системе разностных уравнений относительно значений искомой функции прогиба $\left\{W_{i}\right\}_{i=0}^{N}$ в точках разбиения отрезка $[a ; b]$ на $N$ равных частей с шагом $H=(b-a) / N$. Для цилиндрической части $[a ; b]=\left[x_{0} ; 1\right]$, конической $-[a ; b]=[0 ; 1]$. В каждой из внутренних точек $x_{k}=a+k H, k=\overline{1, N-1}$ отрезка $[a ; b]$ производные от $W$ представляются центральными конечными разностями второго порядка [8]:

$$
W^{\prime}\left(x_{k}\right)=\frac{1}{12 H}\left(-W_{k+2}+8 W_{k+1}-8 W_{k-1}+W_{k-2}\right)+\frac{H^{4}}{18} R_{5} ;
$$




$$
\begin{gathered}
W^{\prime \prime}\left(x_{k}\right)=\frac{1}{12 H^{2}}\left(-W_{k+2}+16 W_{k+1}-30 W_{k}+16 W_{k-1}-W_{k-2}\right)+\frac{H^{4}}{54} R_{6} ; \\
W^{\prime \prime \prime}\left(x_{k}\right)=\frac{1}{2 H^{3}}\left(W_{k+2}-2 W_{k+1}+2 W_{k-1}-W_{k-2}\right)+\frac{17 H^{2}}{60} R_{5} ; \\
W^{(4)}\left(x_{k}\right)=\frac{1}{H^{4}}\left(W_{k+2}-4 W_{k+1}+6 W_{k}-4 W_{k-1}+W_{k-2}\right)+\frac{17 H^{2}}{90} R_{6},
\end{gathered}
$$

где $\left|R_{i}\right|<\max _{x \in\left[l_{0} / l_{1} ; 1\right]}\left|W^{(i)}(x)\right|, i=5 ; 6$.

В граничных условиях производные представляются конечными разностями первого порядка. В случаях жесткого и шарнирного закреплений торцов соответствующе условия можно записать в виде:

$$
\begin{gathered}
W_{0}=0, W_{-1}=W_{1} ; W_{N}=0, W_{N+1}=W_{N-1}, \\
W_{0}=0, W_{-1}=-W_{1} ; W_{N}=0, W_{N+1}=-W_{N-1} .
\end{gathered}
$$

Уравнения (27) позволяют исключить переменные $W_{-1}, W_{N+1}, W_{0}, W_{N}$ в полученной системе. Приравнивание определителя этой системы к нулю приводит к характеристическому уравнению относительно параметров волнообразования $\chi, n$ или $q, n$.

Сопряжение «бочкообразной» $и$ «оживальной» оболочек через шпангоут. Заметим, что для сопряженных оболочек $R=R_{1, \text { cone }}=l_{1} \cos \alpha$. Первоначальное изучение сопряжения оболочек рассматриваемых типов начинается с поиска согласованных значений угловых коэффрициентов касательных обеих оболочек с целью определения значений параметром подъемистости $C_{c y l}$ и $C_{\text {cone }}$. Соответствующее условие согласования имеет вид:

$$
C_{c y l} R \frac{\pi}{L}=\operatorname{ctg} \alpha-C_{\text {cone }} R \frac{\pi}{\left(l_{1}-l_{0}\right) \sin \alpha} .
$$

Приведенные выше ограничения на размеры секций гарантируют положительную определенность обеих частей (28).

Сопряжение оболочек при отсутствующем стыковочном шпангоуте проводится приравниванием значений функции прогиба и ее производных в точке сопряжения. В данном случае имеем:

$$
\begin{gathered}
W_{\text {cone }}(1)=W_{\text {cyl }}(0) ; \quad W_{\text {cone }}^{\prime}(1)=W_{c y l}^{\prime}(0) ; \\
W_{\text {cone }}^{\prime \prime}(1)=W_{c y l}^{\prime \prime}(0) ; \quad W_{\text {cone }}^{\prime \prime \prime}(1)=W_{c y l}^{\prime \prime \prime}(0) .
\end{gathered}
$$


В случае наличия стыковочного шпангоута условие согласования (28) обеспечивает локально конусную форму оболочечной конструкции в окрестности точки сопряжения с углом при «основании» такого конуса

$$
\beta=\operatorname{arctg} \frac{\pi R C_{c y l}}{L} \text { или } \beta=\operatorname{arctg}\left(\operatorname{ctg} \alpha-C_{\text {cone }} R \frac{\pi}{\left(l_{1}-l_{0}\right) \sin \alpha}\right) .
$$

Условия сопряжения секций оболочки через стыковочный шпангоym. Предполагается, что кольцо подкрепляющего шпангоута имеет жесткость на изгиб, как в плоскости начальной кривизны, так и из плоскости. Изменение напряженно-деформированного состояния при переходе через шпангоут на конусе изучено в $[7,8]$, где, в частности, получено:

$$
\begin{gathered}
W_{\text {cone }}(1)=W_{c y l}(0) ; \quad W_{\text {cone }}^{\prime}(1)=W_{c y l}^{\prime}(0) ; \\
W_{\text {cone }}^{\prime \prime}(1)+G_{2} W_{\text {cone }}^{\prime}(1)=W_{c y l}^{\prime \prime}(0) ; \quad W_{\text {cone }}^{\prime \prime \prime}(1)-G_{1} W_{\text {cone }}(1)=W_{c y l}^{\prime \prime \prime}(0), \quad \text { (30) } \\
\text { причем } G_{1}=\frac{n^{4}\left(n^{2}-1\right)^{2}(E J)_{x}^{\text {ring }}}{E h R^{3} \cos ^{3} \beta} ; \quad G_{\text {cone }, 2}^{i}=\frac{n^{2}\left(n^{2}-1\right)^{2}(E J)_{z}^{\text {ring }}}{E h R^{3}\left(n^{2}+1\right)}-\text { без- }
\end{gathered}
$$
размерные жесткости шпангоута; $J_{x}^{\text {ring }}, J_{z}^{\text {ring }}$ - моменты инерции соответственно при изгибе шпангоута в плоскости начальной кривизны и из плоскости.

Модификация системы разностных уравнений при сопряжении оживального и бочкообразного отсеков. Для представления производных в диффреренциальном уравнении (25) (для «оживало») используются конечные разности (26), с такой же нумераций, с шагом $H_{1}=\frac{1-x_{0}}{N}$. В качестве граничных условий выбирается одна из первых пар в соотношениях (27).

В уравнении (23) (для «бочки») конечные разности во внутренних точках отрезка используют нумерацию от $N+4$ до $2 N+2$ и имеют шаг $H_{2}=\frac{1}{N}$. В краевой $(2 N+3)$-ей точке записываются соотношения, аналогичные второй паре из равенств (27). Например, шарнирное закрепление представится равенствами $W_{2 N+3}=0, W_{2 N+4}=-W_{2 N+2}$.

Для того чтобы получить конечные разности для условий сопряжения, применяется формула Тейлора в точках $W_{N}$ и $W_{N+3}$ с приращениями к точкам $W_{N-2}, W_{N-1}, W_{N+1}$ и $W_{N+2}, W_{N+4}, W_{N+5}$ соответственно. Условия (29), (30) представляются в виде:

$$
W_{N}=W_{N+3} \text {; }
$$




$$
\begin{gathered}
\frac{1}{6 H_{1}}\left(W_{N-2}-6 W_{N-1}+3 W_{N}+2 W_{N+1}\right)=\frac{1}{6 H_{2}}\left(-2 W_{N+2}-3 W_{N+3}+6 W_{N+4}-W_{N+5}\right) \\
\frac{1}{H_{1}^{2}}\left(W_{N-1}-2 W_{N}+W_{N+1}\right)+\frac{G_{2}}{6 H_{1}}\left(W_{N-2}-6 W_{N-1}+3 W_{N}+2 W_{N+1}\right)= \\
=\frac{1}{H_{2}^{2}}\left(W_{N+2}-2 W_{N+3}+W_{N+4}\right) ; \\
\frac{1}{H_{1}^{3}}\left(-W_{N-2}+3 W_{N-1}-3 W_{N}+W_{N+1}\right)-G_{1} W_{N}= \\
=\frac{1}{H_{2}^{2}}\left(-W_{N+2}+3 W_{N+3}-3 W_{N+4}+W_{N+5}\right) .
\end{gathered}
$$

Из характеристического уравнения определяется значение числа волн $n$ в окружном направлении, соответствующее наименьшему значению критического давления $q$.

Численные результаты. Рассмотрена оболочечная конструкция с характеристиками: $h_{0}=0,3 \mathrm{~cm}, E=7 \cdot 10^{5} \kappa 2 / \mathrm{cm}^{2}, v=0,32$.

«Оживальная» секция выбирается с параметрами: $l_{1}=182 \mathrm{~cm}, l_{0}=0,45 l_{1}, \quad \alpha=75^{\circ}$, бочкообразная $-L=2,5 R$.

Исследовано влияние относительной подъемистости неподкрепленных «бочки» $C_{c y l}$, «оживало» $C_{\text {cone }}$ (на рис. 2 обозначено $C$ ) и типа закрепления торцов на критическое давление $q$. На рис. 2 абсциссам $C=C_{c y l}=C_{\text {cone }}=0$ соответствуют оболочки без подъема, т.е. соответственно цилиндрической и конической формы.

Равноустойчивость отсеков. Условие согласования (28) выполняется для бесконечного множества коэффициентов $C_{c y l}$ и $C_{c o n e}$. Необходимо выбрать такую пару, для которой выполнено условие равноустойчивости цилиндрического и конического пролетов оболочки, каждая из которых шарнирно оперта. Приближенные значения коэффициентов можно определить, пользуясь графиками рис. 2, а затем уточнить по формуле (28). Так, для оболочечной конструкции с заданными параметрами при значениях

$$
C_{\text {cone }}=0,06258642604 ; \quad C_{c y l}=0,137,
$$

оживальный отсек теряет устойчивость при критическом давлении $q_{\text {rev }}=2,98 \kappa 2 / \mathrm{cm}^{2}$, бочкообразный $-q_{b a r}=2,97 \kappa 2 / \mathrm{cm}^{2}$. При этих же значениях подъемистости расчетная критическая нагрузка составной 
конструкции составляет $q_{\text {constr }}=2,3 \kappa 2 / \mathrm{cm}^{2}$, что на $23 \%$ меньше критических нагрузок, соответствующих равноустойчивости отсеков.

Дальнейшее исследование проведено для конструкции со значениями относительной подъемистости $C_{c y l}$ и $C_{\text {cone }}$, указанными в (31). Для подкрепленной стыковочным шпангоутом конструкции зависимость критического давления $q$ от параметра жесткости шпангоутов $G_{1}$ в плоскости начальной кривизны при фриксированных параметрах жесткости из плоскости начальной кривизны $G_{2}$ приведена на рис. 3 .

Как следует из приведенных зависимостей, с увеличением жесткости шпангоута $G_{1}$ в плоскости начальной кривизны, критическая нагрузка возрастает с дальнейшей стабилизацией зависимости и дальнейшее увеличение параметра $G_{1}$ является нецелесообразным с точки зрения материалоемкости конструкции. Для значений $G_{1}=3000$ и $G_{1}=7000$ критические давления отличаются менее, чем на $2 \%$. Величина параметра $G_{2}$, как показывает численный анализ для рассмотренных параметров исследуемой системы, не приводит к существенному влиянию на критическую нагрузку общей фрормы выпучивания конструкции с захватом стыковочного шпангоута.

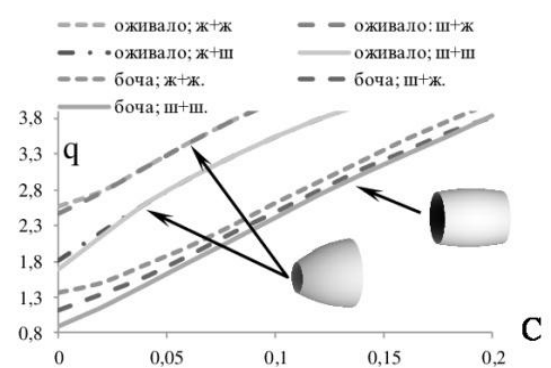

Рис. 2 - Влияние относительной подъемистости образующих конструкций типа «бочка» и «оживало» на величину критической нагрузки при разных условиях закрепления торцов

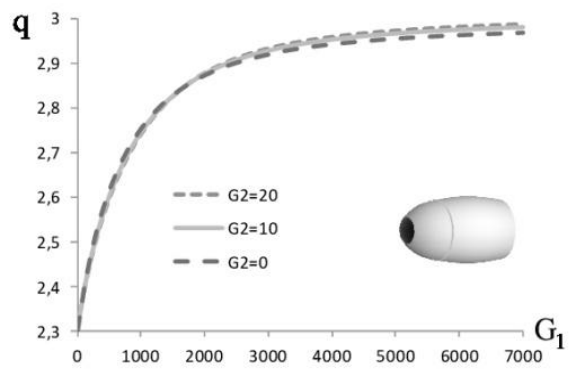

Рис. 3 - Влияние жесткости промежуточного шпангоута в плоскости начальной кривизны и из плоскости на величину критической нагрузки конструкции типа «бочка-оживало»

Как следует из численного анализа (рис. 2), относительный параметр подъемистости отсеков конструкции существенно влияет на вели-

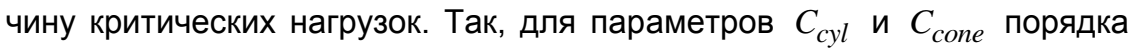
0,1 величина критического давления возрастает более, чем два раза по сравнению с конструкциями без подъемистости образующих.

Достоверность результатов исследования определена предельными переходами к известным соотношениям и сравнением значений критических давлений для отсеков, составляющих исследуемую конструкцию. При отсутствии кривизны образующих отсеков из приведенных 
в работе основных уравнений устойчивости следуют характеристические уравнения для цилиндрической и конической оболочек, а значения критических давлений находятся в соответствии (до 10\%) с результатами работ $[1,3,4,7,8]$, полученными другими методами.

Выводы. Сформулирована постановка задачи устойчивости комбинированной оболочечной конструкции типа «бочка-оживало» при наличии стыковочного шпангоута при действии статического внешнего равномерного давления. Получены основные разрешающие дифференциальные уравнения, позволяющие исследовать конструкцию с учетом радиуса кривизны образующей, жесткостных характеристик стыковочного шпангоута как в плоскости начальной кривизны, так и из плоскости, а также предложен численный алгоритм расчета на базе метода конечных разностей, позволяющий определить величину критических нагрузок локальной и общей фрорм выпучивания при различных условиях закрепления торцов. Указан подход к определению параметров равноустойчивости сопряженной конструкции с выбором рациональной жесткости стыковочного шпангоута.

\section{БИБЛИОГРАФИЧЕСКИЕ ССЫЛКИ}

1. Вольмир А. С. Устойчивость деформируемых систем. М.: Наука, 1967. 984 с.

2. Вышенский В. В., Кузнецов Е. Н., Михайлов П. Д. Тела вращения с минимальным сопротивлением в трансверсальном потоке газа // Ученые записки ЦАГИ. 1992, №2. С. 78-82.

3. Грищак В. З., Маневич А. И. Влияние жесткости шпангоутов на изгиб из плоскости на устойчивость подкрепленной цилиндрической оболочки // Гидроаэромеханика и теория упругости. 1972. Вып. 14. С. 121-130.

4. Дегтяренко П. Г., Грищак В. З., Дьяченко Н. Н. К расчету на устойчивость комбинированной оболочечной конструкции с учетом дискретности расположения промежуточных шпангоутов // Проблеми обчислювальної механіки і міцності конструкцій: зб. наук. праць. 2019. Вип. 29. С. 113-131.

5. Колкунов Н. В. Основы расчета упругих оболочек. М.: Высш. школа, 1972. 296 с.

6. Макаренко И. Н. Устойчивость сопряженных оболочек вращения // Вестн. СПбГУ. Сер.: 1. 2001. Вып. 3 (17). С. 61-69.

7. Печников В. П. Исследование на основе полубезмоментной теории устойчивости конической оболочки, подкрепленной упругими шпангоутами // Изв. вузов. Машиностроение. 1968. № 10. С. 37-42.

8. Преображенский И. Н., Грищак В. З. Устойчивость и колебания конических оболочек. М.: Машиностроение, 1986. 240 с.

9. Хасанов М. М., Чапаев Т. М., Амшоков Б. Х. Устойчивость стенки стального силоса при осесимметричном выпучивании и начальном искривлении оболочки, направленням внутр. // Инж. Вестник Дона. 2018. №2.

URL: http://ivdon.ru/ru/magazine/archive/n2y2018/4944

10. Чапаев Т. М., Балкизов А. Б., Сасиков А. С. и др. Анализ известных теоретических и экспериментальных исследований устойчивости стенки цилиндрического зернохранилища // Инж. Вестник Дона. 2018. №4. URL: http://ivdon.ru/ru/magazine/ archive/n4y2018/5292

11. Zhao $Y$, Teng J. G. A stability design proposal for cone-cylinder intersections under internal pressure // International Journal of Pressure Vessels and Piping, 2003. Vol. 80. No.5. P. 297-309. 


\author{
П. Г. Дегтяренко ${ }^{1}$ В. З. Грищак², д-р техн. наук, \\ Д. Д. Грищак ${ }^{3}$ канд. фріз.-мат. наук, \\ Н. М. Д'яченко ${ }^{2}$ канд.. фріз.-мат. наук
}

\title{
ПОСТАНОВКА ТА ОСНОВНІ РОЗВ'ЯЗУВАЛЬНІ РІВНЯННЯ ЗАДАЧІ СТІЙКОСТІ ОБОЛОНКОВОЇ КОНСТРУКЦІЇХ ТИПУ «БОЧКА-ОЖИВАЛО» ЗА ДІЇ ЗОВНІШНЬОГО ТИСКУ
}

у даній роботі формулюється задача стійкості тонкостінної оболонкової конструкції типу «бочка-оживало» при наявності стикувального шпангоута, що знаходиться під впливом нормального зовнішнього тиску. Отримано новні розв'язувальні диференціальні рівняння, що дозволяють досліджувати конструкцію з урахуванням радіусу кривини твірної і характеристик жорсткості стикувального шпангоута. Розв'язання задачі будується на основі методу скінченних різниць. Виявлено вплив відносної висоти підйому твірних і способів закріплення торців на локальну і загальну стійкість відсіків. Вказано підхід до визначення параметрів рівностійкості сполученої конструкції, а також величини раціональної жорсткості стикувального шпангоута.

Ключові слова: оболонкова конструкція типу «бочка-оживало», локальна та загальна стійкість, жорсткість шпангоута, метод скінченних різниць.

UDC 539.3

P. G. Degtyarenko1, V. Z. Gristchak', Dr. Sci . (Tech.), D.D. Gristchak ${ }^{3}$, PhD (Phys.-Math.), N. M. Dyachenko², PhD (Phys.-Math.)

\section{STATEMENT AND BASIC SOLUTION EQUATIONS OF THE STABILITY PROBLEM FOR THE SHELL-DESIGNED TYPE "BARREL-REVIVED" UNDER EXTERNAL PRESSURE}

In this paper, we formulate the problem of stability of a thin-walled shell design of the "barrel-revived" type in the presence of a docking frame under the influence of normal external pressure. The basic resolving differential equations are obtained that allow one to study the structure taking into account the radius of curvature of the generatrix and the stiffness characteristics of the docking frame. The solution to the problem is based on the finite difference method. The influence of the relative height of the generators and the methods of fastening the ends on the local and general stability of the compartments is revealed. The approach to determining the parameters of equal stability of the conjugate structure, as well as the value of the rational rigidity of the docking frame are presented.

Keywords: "barrel-revived" shell structure, local and general stability, rigidity of the frame, finite difference method.

The issues of rational design of shell systems and reducing their material consumption are most relevant in the construction of new technology, in particular, aviation, chemical, rocket and space and other industries. Recently, special attention of researchers has been directed to the study of effective forms of structural elements that are subject to external force impact from the point of view of bearing capacity and material consumption [2, 9-11]. For example, for aircraft structures and systems of rocket and space tech- 
nology, it seems relevant to study smooth and orthogonal reinforced ribs of the shells of revolution in the presence of compartments of different geometric shapes, the most effective from the point of view of their stability when exposed to external loads and aerodynamic characteristics.

This work is devoted to the formulation of the statement of the problem and the derivation of the basic stability equations of a composite shell system of the "barrel-revived" type. The main attention is paid to the determination of critical loads for local and general buckling forms under the action of uniform external pressure in the presence of a docking frame. To take into account changes in the stress-strain state of the structure during the transition through the frame, the research results presented in $[3,4,6-8]$ were used. In accordance with [1,5], it is assumed that the relative lift of both compartments of the structure is less than one fifth of its smallest linear size, which allowed approximate calculation of the generally accepted coordinate lines for cylindrical and conical shells as coordinate lines, respectively. A numerical calculation algorithm based on the finite difference method is proposed, which allows one to determine the critical loads under various boundary conditions. The approach to determining the parameters of equal stability of the coupled structure with the choice of the rational rigidity of the docking frame is presented. In the limiting cases considered, the critical pressures are in agreement (up to 10\%) with the results of $[1,3,4,7,8]$ obtained by other methods.

\section{REFERENCES}

1. Volmir A. S. Stability of deformable systems. Moscow: Nauka, 1967. 984 p. (in Russian).

2. Vishensky V. V., Kuznetsov E. N., Mikhailov P. D. Bodies of rotation with minimal resistance in a transverse gas flow // Scientific notes of TsAGI. 1992, No. 2. P. 78-82 (in Russian).

3. Gristchak V. Z., Manevich A.I. Influence of a ring stiffness on a bend out of a plane on the stability of a reinforced cylindrical shell // Gidroaeromekhanika i teoriya uprugosti. 1972. Issue 14. P. 121-130 (in Russian).

4. Degtyarenko P. G., Gristchak V. Z., Dyachenko N. N. To the stability calculation of a combined shell structure taking into account the discreteness location of the intermediate rings // Problems of computational, mechanics and strength of structures. 2019. Vol. 29. P. 113-131 (in Russian).

5. Kolkunov N.V. Basics of calculating elastic shells. Moscow: Vyisshaya shkola, 1972. 296 p. (in Russian).

6. Makarenko I.N. Stability of conjugated rotation shells // Vestnik St.Petersburg University. Ser. 1. 2001. Iss. 3 (17). P. 61-69 (in Russian).

7. Pechnikov V. $\boldsymbol{P}$. Investigation on the basis of a semi-momentless theory of the stability of a conical shell reinforced by elastic rings // Izv. vuzov. Mashinostroenie. 1968. No10. P. 37-42 (in Russian).

8. Preobrazhensky I. N., Gristchak V. Z. Stability and oscillations of conical shells. Moscow: Mashinostroenie. 1986. 240 p. (in Russian). 
9. Khasanov M. M., Chapaev T. M., Amshokov B. Kh. The stability of the wall of a steel silo with axisymmetric buckling and initial curvature of the shell directed inward // Inženernyj vestnik Dona. 2018, No. 2.

URL: http://ivdon.ru/ru/magazine/archive/n2y2018/4944 (in Russian).

10. ChapaevT. M., BalkizovA. B., SasikovA. S. and oth. Analysis of known theoretical and experimental studies of the stability of the wall of a cylindrical granary // Inženernyj vestnik Dona. 2018, No. 4. URL: http://ivdon.ru/ru/magazine/archive/n4y2018/5292 (in Russian).

11. Zhao $Y$, Teng J. G. A stability design proposal for cone-cylinder intersections under internal pressure // International Journal of Pressure Vessels and Piping, 2003. Vol. 80. No 5. P. 297-309.

${ }^{1}$ Державне космічне агентство України, Київ, Україна;

${ }^{2}$ Запорізький національний університет, Запоріжжя, Україна;

${ }^{3}$ Центральний науково-дослідний інститут озброєння та військової техніки Міністерства оборони України, Київ, Україна 